\title{
Ultrasound imaging to localize foramina for superficial trigeminal nerve block
}

\author{
Ban C. H. Tsui, MD
}

Received: 14 May 2009/ Accepted: 26 May 2009/Published online: 6 June 2009

(C) Canadian Anesthesiologists' Society 2009

\section{To the Editor,}

Superficial trigeminal nerve blocks are performed by injecting local anesthetic in close proximity to the three individual terminal superficial branches of the trigeminal nerve divisions; i.e., supraorbital (of ophthalmic nerve; V1 division), infraorbital (of maxillary nerve; V2 division), and mental (sensory terminal branch of mandibular nerve; V3 division). Each nerve is anatomically close to its respective foramen (Fig. 1); thus nerve localization for this block ideally relies on a landmark-based approach using palpation of the foramina. However, occasionally it can be challenging to identify each foramen by relying on palpation alone. In these cases, many experts "search" for the foramina by gently probing with a small-gauge needle.

Ultrasound (US) imaging is a safe simple non-invasive modality through which soft tissues and nerve structures can be visualized and identified when combined with a thorough knowledge of regional anatomy. However, there is limited information on the use of US for the identification of bony structures. Definitive identification of intraosseous landmarks, e.g., a tubercle, foramen, or notch around or through which the nerve travels, may be particularly important when, due to its small size and/or imaging artefacts, the target nerve of the block is unidentifiable with US. Sonographically, bone appears as a hyperechoic linear edge (white line) with an underlying anechoic (dark) shadow. Any disruption within the hyperechoic line may indicate either an anatomical defect or a discontinuity in the bone, such as a foramen.

B. C. H. Tsui, MD ( $\square)$

University of Alberta, Edmonton, AB, Canada

e-mail: btsui@ualberta.ca

URL: www.EdmARA.ca
Using the above concept of discontinuity in the bone, we describe here a simple US-guided approach to locate the three important landmark foramina for superficial trigeminal nerve block. Using a high-frequency linear transducer (e.g., SLA 6-13 MHz 25 mm footprint hockeystick probe, MicroMaxx ${ }^{\circledR}$, Sonosite, Bothell, WA, USA), the foramina can be identified dynamically by subtle movement of the probe position after its placement in the usual vicinity of the foramina in order to capture the accompanying break in the linear hyperechogenicity of the bony surface.

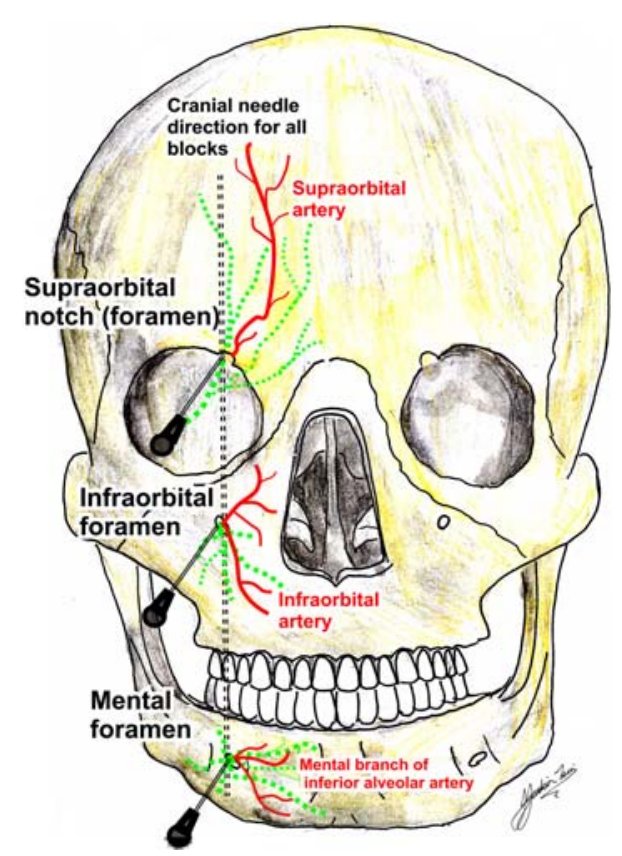

Fig. 1 Schematic diagram showing the bony landmarks (foramina), target nerves, and needle insertion for trigeminal nerve block. Dotted lines depict nerves; solid lines depict arteries 
Fig. 2 Ultrasound images

using colour Doppler with

schematic overlay of probe

scanning techniques, where

scanning is in the direction

from A to B. (Top) Supraorbital

notch/foramen and artery;

(middle) infraorbital foramen

and artery; (bottom) mental

foramen
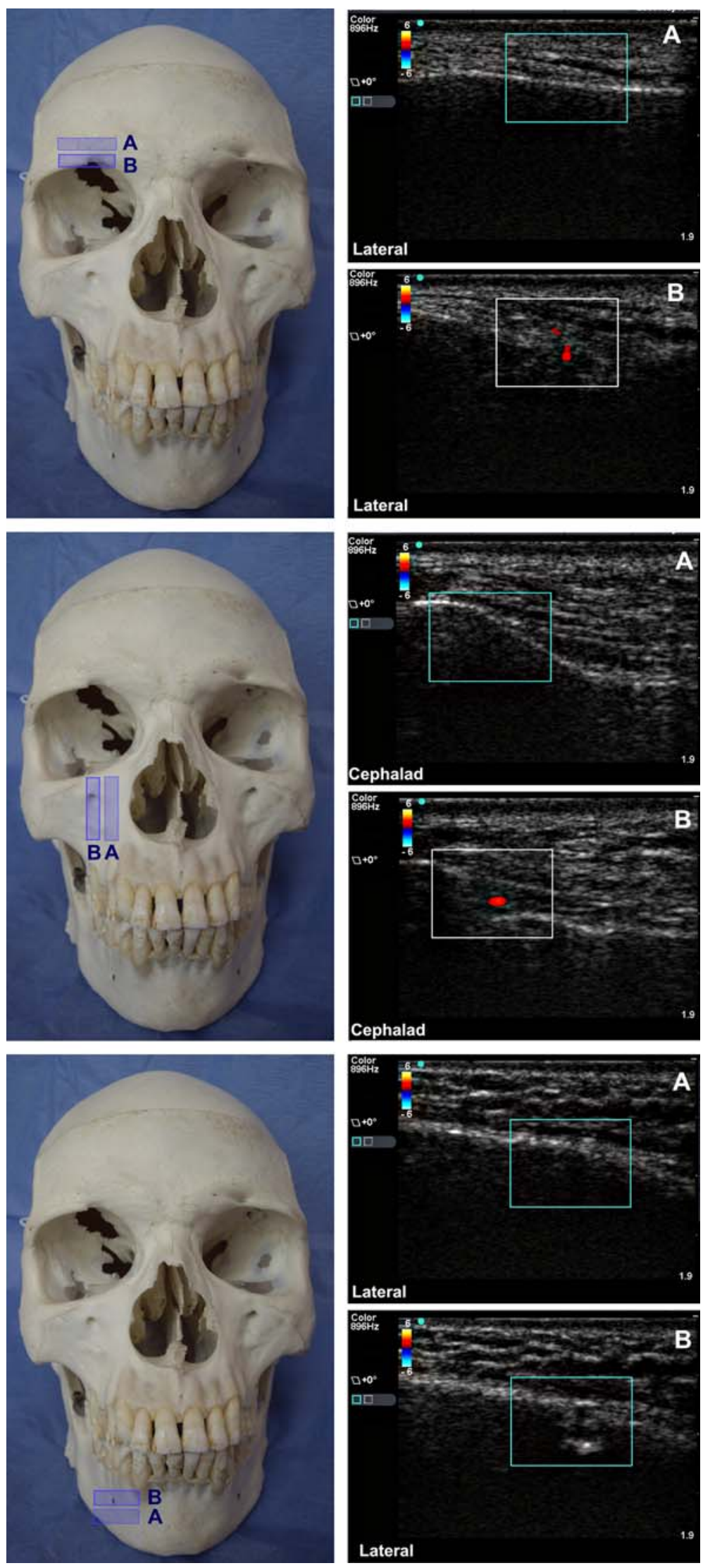
All foramina typically lie in the corresponding sagittal plane on each side of the face (Fig. 1). In adults, all three foramina are located approximately $2.5 \mathrm{~cm}$ lateral to the midfacial line passing through the pupil. To localize the supraorbital notch (foramen), the probe is positioned transversely above the roof of the orbital rim and in alignment with the pupil, and the bone is scanned slowly in a cephalad-to-caudad direction (Fig. 2). The infraorbital foramen is generally located about $1 \mathrm{~cm}$ below the middle of the lower orbital margin (inferior rim of orbit) and can be readily located by scanning in a sagittal plane from medial to lateral along the lower orbital margin. In children, the infraorbital foramen can often be palpated easily, with the exception of neonates due to their developing facial configuration. For pediatrics, a simple mathematical formula has been developed (distance from the midline $=$ $21 \mathrm{~mm}+0.5 \times$ age [in years] $)^{1}$ and may be useful when localizing the foramen either blindly or with US. Finally, the mental foramen, which lies inferior to the outer lip at the level of the second premolar, midway between the upper and lower borders of the mandible, ${ }^{2}$ is localized using a transverse plane and scanning in a cephalad direction from the inferior border of the mandible. During this dynamic scanning, the image of the bony surface will change from a definite linear border to a border with a hypoechoic break indicating the foramen. In addition, the identity of each orifice can be verified by illumination of their contained blood vessels using colour Doppler. The images in Fig. 2 were obtained using colour Doppler and highlight the identity of the supra and infraorbital arteries. Although the mental branch of the inferior alveolar artery exits from the mental foramen, it has been difficult to illuminate this artery in our experience, perhaps due to the artery's relatively small size. With the aforementioned imaging techniques, clear views of the three distinct openings of the respective foramina can be obtained with ease, thus supporting a potential role for imaging to facilitate trigeminal nerve blocks. Further study is needed to examine the merit of using US to identify foramen as anatomical landmarks for nerve localization when performing superficial trigeminal nerve blocks. This letter serves to remind the reader that US is not only useful for soft tissue and nerve structure recognition, but it can also be valuable for identifying bony structures in situations where nerve visibility is poor or unattainable.

Acknowledgement The author thanks Mr. Jenkin Tsui for his original simplified anatomical drawing and for permission to use his artwork.

Financial support Dr. Tsui's work is supported in part by a Clinical Scholar Award from the Alberta Heritage Foundation for Medical Research.

Conflicts of interest None declared.

\section{References}

1. Suresh S, Voronov $P$, Curran J. Infraorbital nerve block in children: a computerized tomographic measurement of the location of the infraorbital foramen. Reg Anesth Pain Med 2006; 31: 211-4.

2. Voronov $P$, Suresh $S$. Head and neck blocks in children. Curr Opin Anaesthesiol 2008; 21: 317-22. 